\title{
Spatially Resolving Lithiation in Silicon-graphite Composite Electrodes via in situ High-energy X-ray \\ Diffraction Computed Tomography
}

Donal P. Finegan ${ }^{1 *}$, Antonis Vamvakeros ${ }^{2,3}$, Lei $\mathrm{CaO}^{1}{ }^{1}$, Chun Tan ${ }^{4,5}$, Thomas M.M. Heenan ${ }^{4,5}$, Sohrab R. Daemi ${ }^{4}$, Simon

D. M. Jacques ${ }^{3}$, Andrew M. Beale ${ }^{3,6,7}$, Marco Di Michiel ${ }^{2}$, Kandler Smith ${ }^{1}$, Dan J.L. Brett ${ }^{4,5}$, Paul R. Shearing ${ }^{4,5 * *}$, Chunmei Ban ${ }^{1 * * *}$

1. National Renewable Energy Laboratory, 15013 Denver W Parkway, Golden, CO 80401, USA

2. ESRF-The European Synchrotron, 71 Avenue des Martyrs, 38000 Grenoble, France

3. Finden Limited, Merchant House, 5 East St Helens Street, Abingdon, OX14 5EG, UK.

4. Electrochemical Innovation Laboratory, Department of Chemical Engineering, University College London, London, WC1E 7JE, UK

5. The Faraday Institution, Quad One, Harwell Science and Innovation Campus, Didcot, OX11 ORA, UK

6. Department of Chemistry, 20 Gordon Street, University College London, London, WC1H OAJ, UK

7. Research Complex at Harwell, Harwell Science and Innovation Campus, Rutherford Appleton Laboratories, Harwell, Didcot, Oxon, OX11 OFA, UK

Corresponding authors:

\begin{abstract}
Optimizing chemical and morphological parameters of lithium-ion (Li-ion) electrodes is extremely challenging; in part due to the absence of techniques to construct spatial and temporal descriptions of chemical and morphological heterogeneities. We present the first demonstration of combined high-speed X-ray diffraction (XRD) and XRD computed tomography (XRD-CT) to probe, in 3D, crystallographic heterogeneities within Li-ion electrodes with a spatial resolution of $1 \mu \mathrm{m}$. The local charge transfer mechanism within, and between individual particles was investigated in a silicon-graphite composite electrode. High-speed XRD revealed charge balancing kinetics between the graphite and Si during the minutes following the transition from operation to open-circuit. Sub-particle lithiation heterogeneities in both Si and graphite were observed using XRD-CT, where the core and shell structures were segmented, and their respective diffraction patterns characterized.
\end{abstract}


Key words: Composite electrodes, lithitation heterogeneities, Si electrodes, X-ray diffraction computed tomography (XRD-CT), high-speed XRD

\section{Main text}

The majority of mechanisms that lead to degradation and performance loss of Li-ion cell electrodes can be characterized by chemical, crystallographic and morphological heterogeneities that are present or develop during operation ${ }^{1}$. Particle and electrode cracking can arise from heterogeneous strains present in the active material or electrode matrix. The morphological evolution of electrode particles during operation can lead to the development of local strains ${ }^{2}$, causing particle cracking and electrode delamination. Non-uniform particle sizes and spatial distributions of conductive carbon within an electrode can lead to some regions of the electrode being underutilized or degrading at an accelerated rate relative to the bulk. Understanding the link between cell performance and dynamic chemical, crystallographic, and morphological heterogeneities within electrodes is paramount to engineering cells that are optimized for specific operating conditions.

Spectroscopy techniques that have previously been used to explore chemical and architectural dynamics of Li-ion electrode materials have included X-ray absorption near-edge spectroscopy (XANES) ${ }^{3,4}$, transmission X-ray microscopy $(\mathrm{TXM})^{5,6}, \operatorname{Raman}^{7}$, and micro-diffraction ${ }^{8,9}$. These techniques have facilitated in situ and operando quantification of lithium concentration gradients ${ }^{9-11}$ and strains ${ }^{12-16}$ within electrode particles. X-ray techniques are now widely used for probing chemical and morphological dynamics in electrode materials across multiple length scales. For example, the evolution of lithiation fronts and crack propagation have been tracked via X-ray computed tomography $(\mathrm{CT})^{17,18}$, and operando X-ray CT in combination with image correlation techniques have been used to map local displacements within Si and Si-graphite electrodes ${ }^{19,} 20$ where highest strains were seen to form around Si particles that exhibit the greatest volumetric expansion during lithiation. For most commercially relevant materials, 
such as graphite and transition-metal oxides, spatially mapping lithiation and chemical degradation requires techniques that are highly sensitive to changes in the crystal structure or chemical composition ${ }^{6}$, 21.

X-ray diffraction (XRD) has been extensively used to characterize the phases of intercalation materials, such as graphite, ex- or in situ $u^{22,23}$. Early in situ experiments ${ }^{22}$ involved scans that were carried out while the cell was at open circuit, had long exposure times, and relied on bulk electrode measurements. With the advancement of high-flux synchrotron sources, focusing optics and detectors, high-resolution point scans are now feasible in short amounts of time, facilitating spatially-resolved operando diffraction measurements ${ }^{11,24}$. Synchrotron XRD has been used to monitor the activity of distinct electrode chemistries in layered composite electrodes and to construct depth profiles of lithiation within a $\mathrm{Si}$ electrode, by recording point scans from separator to current collector and monitoring the phase change from crystalline Si to amorphous phase during its first lithiation 2025 . Using XRD-CT to identify 3D crystallographic heterogeneities in the bulk electrodes has recently been demonstrated in Ni-metal hydride and sodium-ion cells $s^{26-28}$. Sub $1 \mu \mathrm{m}$ XRD-CT scans have been achieved but acquisition times have been on the order of many hours ${ }^{29,30}$. In this work, we leveraged the time-resolved, high-resolution XRDCT capabilities at Beamline ID15A at The European Synchrotron ${ }^{31,32}$ to investigate dynamic processes in 3D with micron-scale resolution.

A Si-graphite composite electrode has been selected for this research to identify the spatial heterogeneity in structure and morphology because of the chemical interaction between these two components. The Sigraphite composite electrode consists of two materials. Both are electrochemically active and can reversibly host lithium at different voltages. Electrochemical interaction between these two active materials is expected during operation. Silicon $(\mathrm{Si})$ is an attractive electrode material for high-energy density Li-ion cells, having a theoretical specific energy density > 10x that of graphite $\left(4200 \mathrm{mAh} \mathrm{g}^{-1}\right.$ for Si 
compared to $372 \mathrm{mAh} \mathrm{g}^{-1}$ for graphite) ${ }^{33,34}$. Optimization of such composite electrodes with respect to composition $^{35}$, active material distribution, and morphological parameters is particularly challenging due to the inherent complexity of interactions between materials with different electrochemical and mechanical properties. Charge imbalances have been shown to accrue during operation of Li-ion electrodes leading to ion transfer between particles at open circuit, which is known as a relaxation period $^{36-38}$. For composite electrodes, this phenomenon of charge-imbalance is expected to be even more severe and can significantly affect the cycling performance ${ }^{39}$. The composite electrode is therefore a model sample for understanding the dynamic heterogeneity between the electrode and the individual particles.

Here, XRD measurements are used to explore charge-balancing kinetics following the transition from operation to open circuit. Sub-particle chemical heterogeneities are also investigated with a resolution of $1 \mu \mathrm{m}$ for 3D XRD-CT imaging for both $\mathrm{Si}$ and graphite phases. Spatial heterogeneities within single electrode particles (Si and graphite) were identified ${ }^{40,41}$. This demonstrates a significant step forward in spatially and temporally resolving states of charge and degradation phenomena within electrode particles during operation and is expected to be a platform study for further investigations into chemical heterogeneities in operating electrochemical cells.

To assess the performance of the Si-graphite composite electrode, the electrode was cycled inside two coin cells with Si-graphite working electrode and lithium metal as the counter electrode. Details on the manufacture of the cell are provided in the Experiment section. The cells were discharged (lithiated) at $0.257 \mathrm{~mA}$ (ca. C/20) to $0.01 \mathrm{~V}$ and charged (delithiated) to $1 \mathrm{~V}$ at $0.257 \mathrm{~mA}$ and held until the current was less than $0.032 \mathrm{~mA}$. The voltage responses of the cells are shown in Figure 1a. The voltage profile of the first lithiation process is quite different from that of the second lithiation process, primarily due to the phase transformation of the Si component as well as the formation of the solid electrolyte interphase. 
High capacity degradation is observed for both cells, which is typical for the Si-based electrodes, as shown in Figure 1b. Notably, the capacity fade following the first cycle was $13.8 \%$.
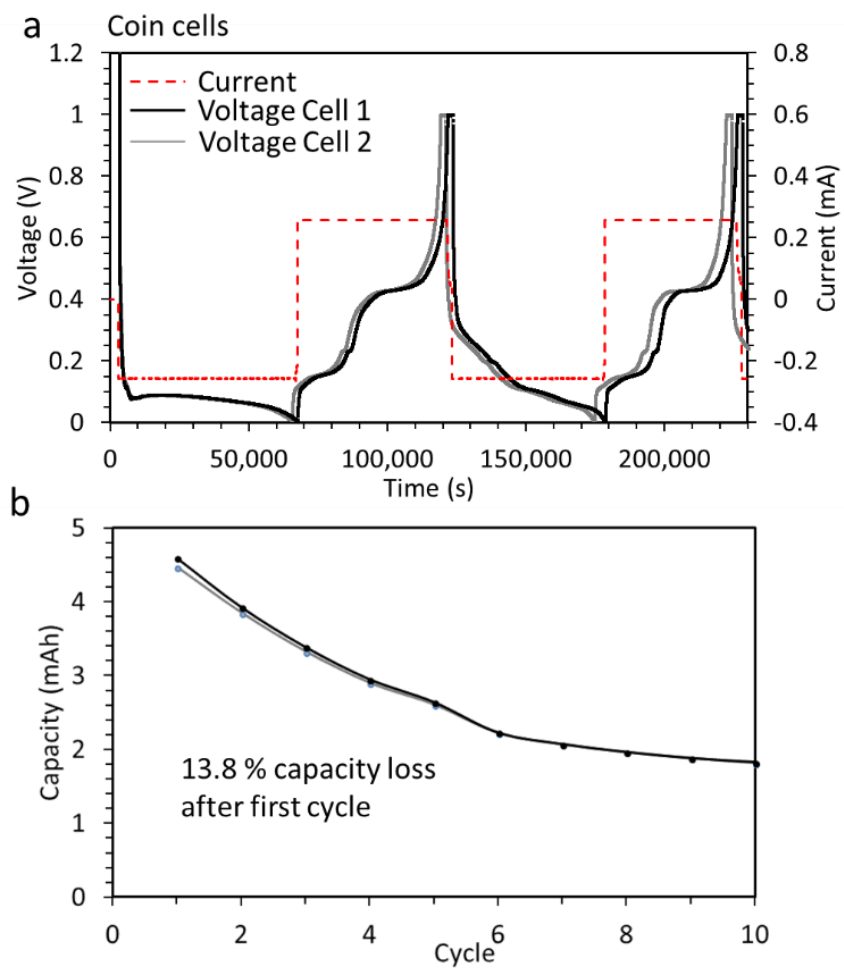

Figure 1. Si-graphite composite electrode performance. a) Cycling plots from the first two cycles of two Li vs Si-graphite composite electrode cells in a coin-cell format, and b) their respective capacity loss over the first ten cycles.

An in-house designed micro-cell was used to conduct the operando characterization ${ }^{42}$, as detailed in the Experimental section and shown in Figure 2a. The Si-graphite composite electrode material was first inserted into the micro-cell and tested without exposure to X-rays. The cell underwent discharging and charging at $2 \mu \mathrm{A}(\mathrm{C} / 16)$, followed by a $4 \mu \mathrm{A}(\mathrm{C} / 8)$ discharge/charge cycle. The voltage profile of the microcell is shown in Figure 2b. As described in the Experimental section, the Si-graphite electrode was inserted in the middle of the customized cell, with a diameter of $1.2 \mathrm{~mm}$ (shown in Figure 2c). The voltage profile 
for the first cycle confirms the normal electrochemical activity from both graphite and silicon active components. To ensure the electrochemical behavior of the Si-graphite electrode in the micro-cell, the cell was tested under higher rates, $C / 16$, later $C / 8$-as compared to $C / 20$ used in the coin cell. The lower capacity, as shown in the Figure $1 \mathrm{~b}$, is expected when using higher cycling rates. The voltage profile from the customized micro-cell confirms the reliable electrochemical responses from both active components.

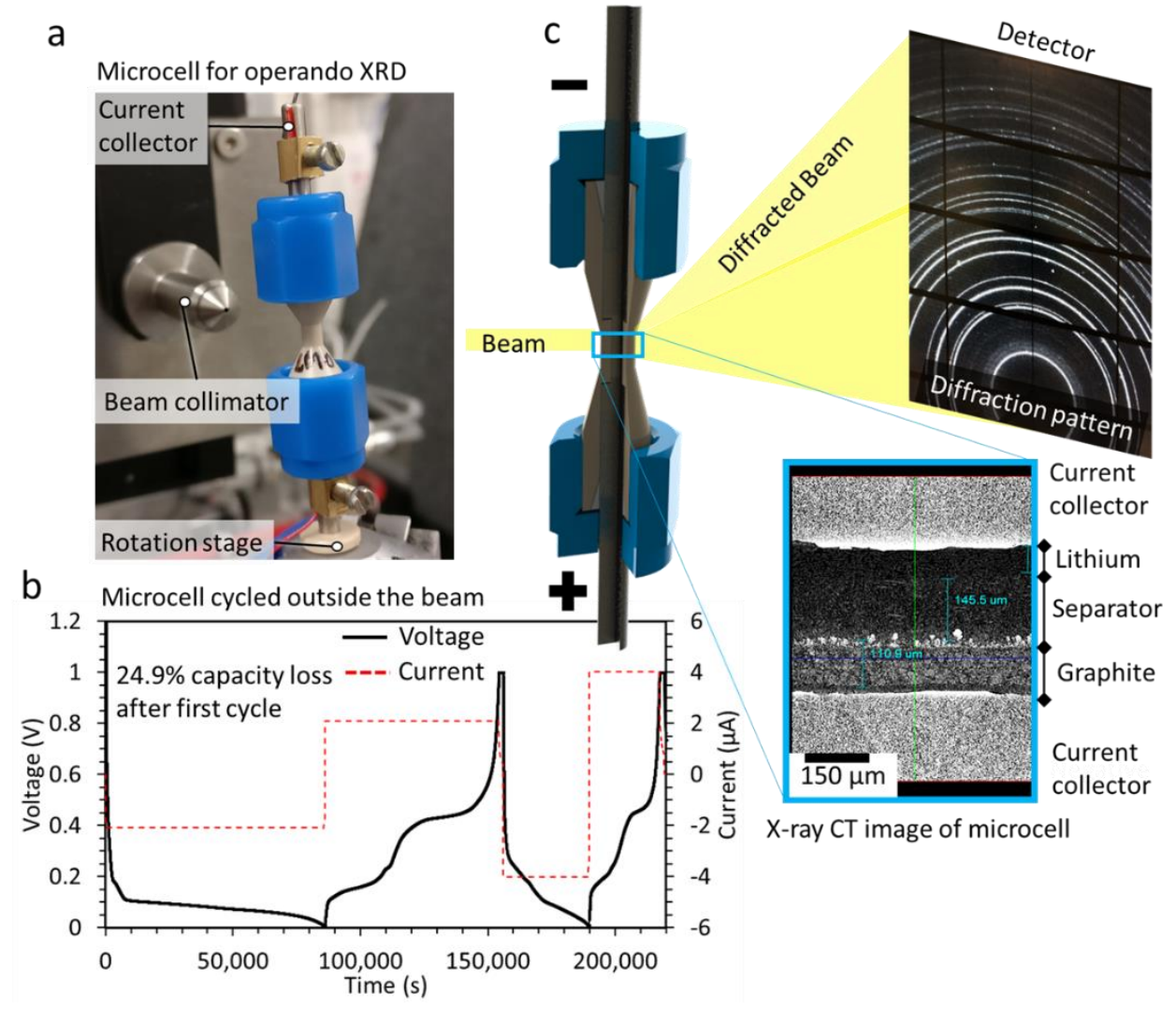

Figure 2. X-ray compatible micro-cell. a) Photograph of the micro-cell in place before an operando XRD experiment. b) Cycling plot and capacity loss after first two cycles of electrode in micro-cell format. In both cases, Li vs Si-graphite composite cells were used, and cycling was performed outside the X-ray beam. c) Cross-sectioned illustration of the micro-cell showing an example of a diffraction profile on the detector and an X-ray CT slice of the internal cell. 
Before placing the micro-cell in the beam for operando imaging, it was discharged at $\mathrm{C} / 15$ to $0.01 \mathrm{~V}$. The cell was charged in steps that were separated by periods of open circuit to investigate activity that occurred following operation of the composite electrodes. During operando XRD measurements, the micro-cell was charged at $5 \mu \mathrm{A}(\mathrm{ca} . \mathrm{C} / 6.3)$ up to $1 \mathrm{~V}$ with 20 min charge steps followed by 16 mins of opencircuit, during which high-speed XRD was carried out (Figure 3a). The total charge time was 4.7 hours, amounting for ca. $74 \%$ of the electrode's capacity. During the experiment, the X-ray detector unexpectedly stopped recording data, a period that is marked by the grey bar in Figure 3a. The electrochemical response of the cell during the experiment (Figure 3a) did not match its behavior during cycling outside the beam (Figure $2 \mathrm{~b}$ ) as it had a more continuous increase in voltage rather than exhibiting the characteristic step increase of graphite. This is thought to be due to a combination of the incident high-energy beam inducing ionization along the path of the beam, as well as less reliable pressure asserted on the cell by the current-collecting pins $^{43}$. It is also possible that the sample was heated by the beam and that pins were not as well compressed as they were for Figure $2 \mathrm{~b}$.

The charge process involved delithiation of the Si-graphite electrode. As seen in the diffractograms from the bulk electrode in Figure $3 b$, the crystalline Si peaks around $Q=2 \AA^{-1}$ diminish initially as the Si phase largely becomes amorphous or semi-crystalline upon lithiation. The $\mathrm{LiC}_{12}$ peaks appear to be consistent in Figure $3 b$, but small shifts were identified through Rietveld refinement and were used to determine its lithiation stage. 

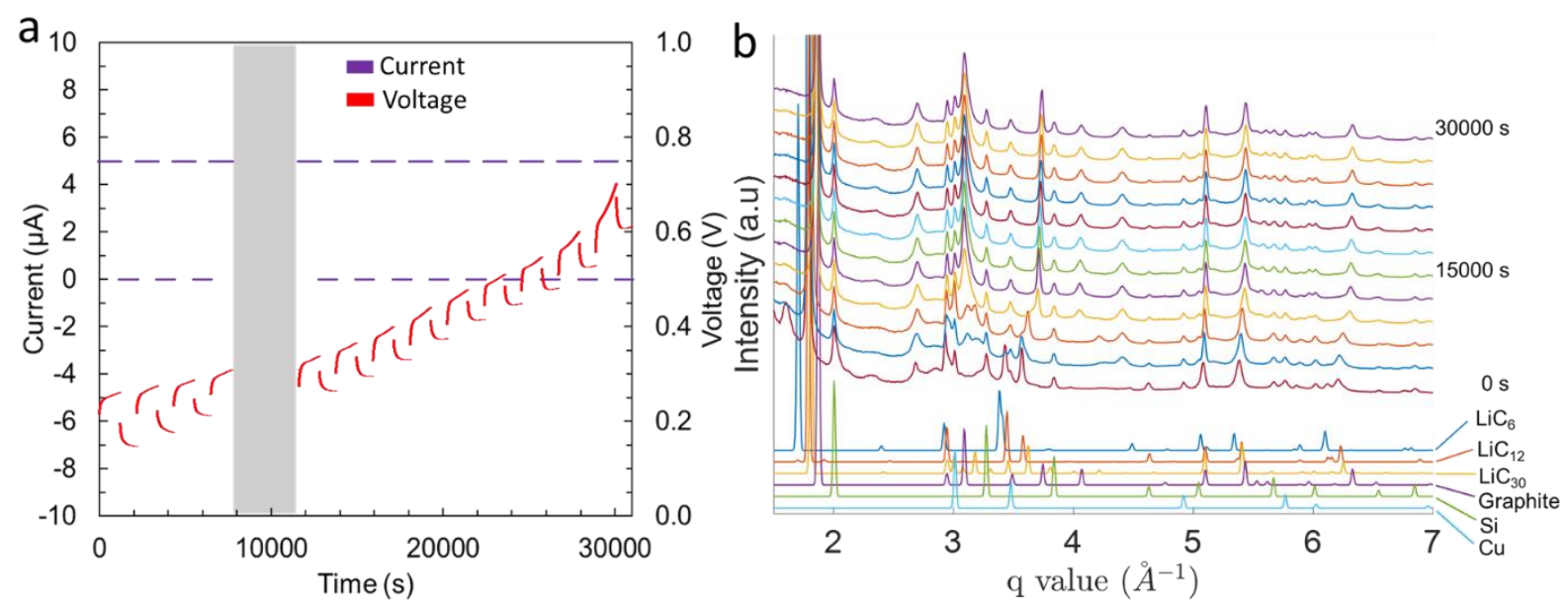

Figure 3. Voltage and diffraction profiles for the micro-cell. a) Voltage and current profile of the microcell during operando XRD measurements where the cell was repeatedly operated for 20 mins followed by open-circuit for 16 mins. The grey region is where the detector crashed, and data was not recorded. b) Diffraction profiles taken during the experiment from beginning to end showing the slight shift and change of peaks. Characteristic diffraction profiles of $\mathrm{Cu}, \mathrm{Si}$, graphite, $\mathrm{LiC}_{30}, \mathrm{LiC}_{12}$ and $\mathrm{LiC}_{6}$ are also included for reference.

The XRD imaging sequence consisted of an XRD-CT scan at the beginning of the charge process, followed by high-speed XRD point scans at $0.1 \mathrm{~Hz}, 100 \mathrm{~Hz}$, and $10 \mathrm{~Hz}$ during operation and subsequent open-circuit, and final XRD-CT scan upon completion of the charge step. The different point-scan XRD frequencies were chosen to capture, in detail, the electrochemical activity that occurred upon transitioning from operation to open-circuit. During operation, point scans were carried out at $0.1 \mathrm{~Hz}$, then point scans were carried out at $100 \mathrm{~Hz}$ for the first $15 \mathrm{~s}$ of open circuit, followed by $10 \mathrm{~Hz}$ for the remaining 16 mins of open circuit.

The intercalation stages of $\mathrm{Li}-\mathrm{C}$ refer to the approximate number of graphene layers between lithium layers and was first proposed by Daumas and Hérold ${ }^{44}$, 45. The first stage of $\mathrm{Li}_{x} \mathrm{C}_{6}(x<0.04)$ is referred to as dilute stage $1^{22}$. Thereafter the stages successively transition from stage $4\left(\mathrm{LiC}_{24}\right)$, to stage $3\left(\mathrm{LiC}_{18}\right)$, stage $2\left(\mathrm{LiC}_{12}\right)$, and finally stage $1\left(\mathrm{LiC}_{6}\right)^{22,46,47}$. Between the stages, 'liquid' ( $\left.\mathrm{L}\right)$ phases exist where $\mathrm{Li}$ is miscible 
with distinct phases leading to some disorder. For example, stage $1 \mathrm{~L}$ carries graphite order and some disorder due to lithium insertion at room temperature ${ }^{48}$. Each transition between stages involves a fixed change in the potential of lithiated graphite that is observed as a plateau in the voltage curve of graphite vs lithium cells. The transition mechanisms between phases are still under debate.

Here, the Li-C phase transitioned from a $\mathrm{LiC}_{12}$ (stage 2) with $d$-spacing values in the region of $3.50 \AA$, to graphite with $d$-spacing of $3.35 \AA^{39,49}$, as shown in Figure 4 a. The quality of fit for $\mathrm{LiC}_{12}$ and graphite using Rietveld refinement is provided in Section 1 of Supplementary Material. A continuity in the $d$-spacing between the $\mathrm{LiC}_{12}$ and graphite phases is observed in Region 1 of Figure 4a rather than distinct phase transitions during the delithiation step. Four distinct diffraction peaks were identified during delithiation from the $\mathrm{LiC}_{12}$ phase (Section 2 of Supplementary Material). The areas of the four peaks were measured, normalized with respect to the total peak area, and plotted as a function of time in Figure $4 \mathrm{~b}$, showing similar results to those found by Yao et al. ${ }^{24}$. Between $\mathrm{LiC}_{12}$ and graphite, there are distinct phases along with liquid phases, e.g. $\mathrm{LiC}_{18}$ and $\mathrm{LiC}_{30} .{ }^{50} \mathrm{Here}$, some peaks observed did not have a stable peak position but dynamically cover a range of peak positions during delithiation. These phases can be considered to be liquid phases, hence the labelling of normalized peak area in Figure 4b. It is observed that multiple phases co-exist. This leads to a continuity of $d$-spacing values during delithiation as opposed to a step change that would otherwise be associated with a transition from one phase to another i.e. the 'measured' or determined $d$-spacing (albeit accurately determined by Rietveld) presented in Figure 4a thus represents the systems structural average.

It was also considered that the expansion of Si particles may have applied force on the interstitial graphite leading to lattice strain. However, there was no noticeable strain observed $(>0.05)$ in the graphite phase (see Section 2 of Supplementary Material); the thickness of the separator in this case $(100 \mu \mathrm{m})$ is expected to have facilitated some flexibility to mitigate compressive affects. For example, it has been demonstrated 
that for graphite electrodes, expansion of particles is expected to reduce porosity rather than apply significant strain on neighboring particles ${ }^{2}$. From Region 2 onwards in Figure 4a, only a single peak existed which corresponded to the solid-solution graphite phase. Consequently, for Region 2 we could carry out Rietveld refinement and calculate the lattice parameter for the graphite phase (Figure 4c). 

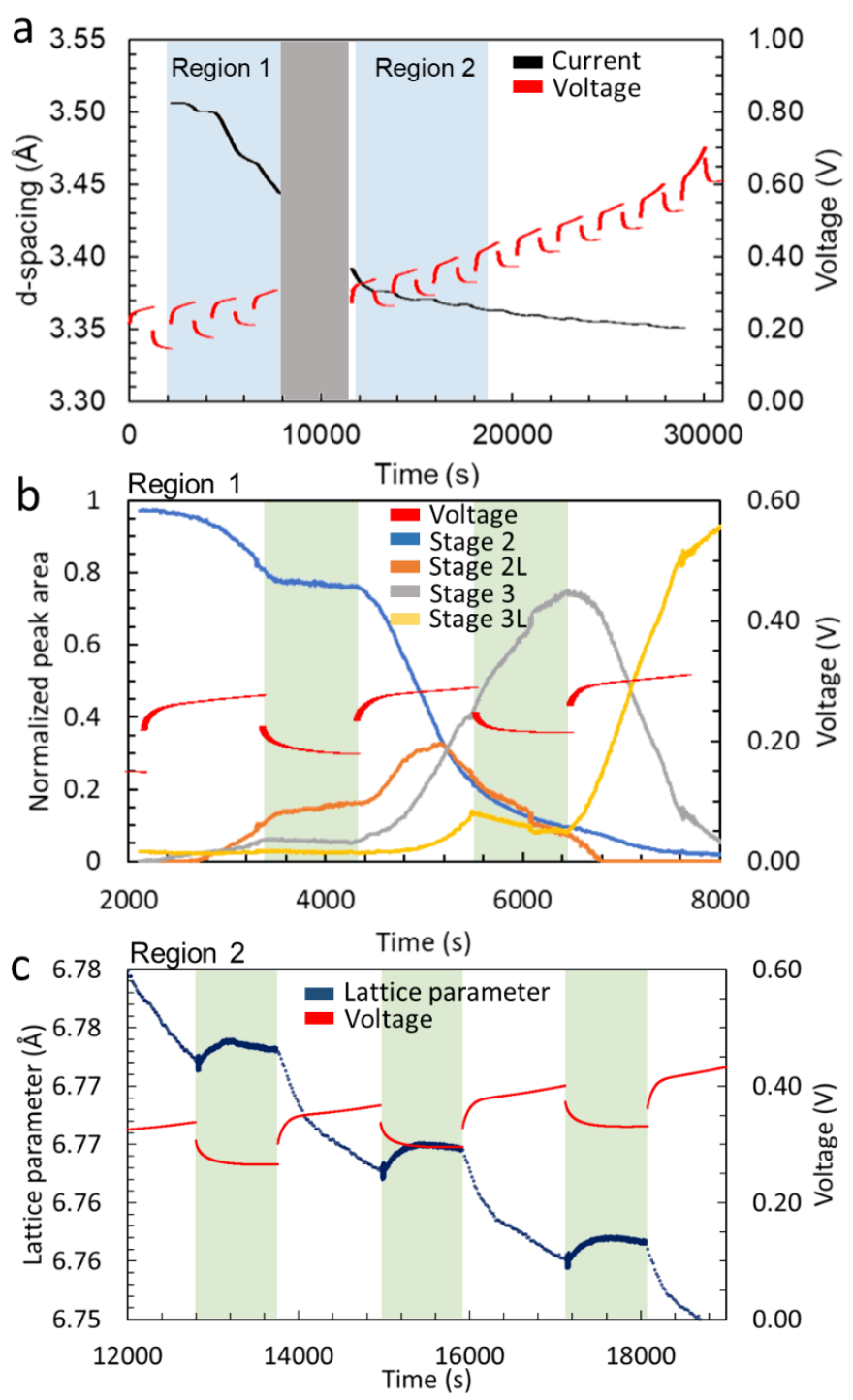

Figure 4. Charge profile and corresponding phases. a) $d$-spacing of the (002) reflection of the $\mathrm{Li}_{x} \mathrm{C}_{6}$ structure during operation and open-circuit periods with blue highlighted regions of interest, and a grey region that marks the time when the detector crashed. XRD-CT slices were captured at the beginning and end of the charge step. (b) Magnified time period of Region 1 showing the integrated peak areas of the $\mathrm{Li}_{x} \mathrm{C}_{6}$ stages during delithiation. The green regions are open-circuit. (c) Magnified region of interest showing the c-lattice parameter of the solid-solution graphite phase during operation and open-circuit (green), where approximately $0<x<0.1$ in $\mathrm{Li}_{x} \mathrm{C}_{6}$. 
From Figure 4 , it is observed that small changes of $x$ in $\mathrm{Li}_{x} \mathrm{C}_{6}$ followed the transition from operation to open-circuit which demonstrate equilibration kinetics outside of operation. This is expected to have been due to the graphite and Si phases having different electrochemical potentials during operation and equilibrating during cell relaxation at open circuit. The open circuit periods are highlighted in green in Figure 4b,c. As observed in Region 1 (Figure 4b), delithiation initially occurred at open circuit, as observed by a continued change to stages of lower lithiation. When the electrode transitioned to the graphite phase in Region 2, the graphite lithiated at open circuit as observed by an increase in the lattice parameter in Figure 4c. Delithiation in the Region 1 and lithiation in Region 2 is likely to have been caused by a crossover in the respective electrochemical potentials of graphite and Si vs Li. For example, at a high state of lithiation the electrochemical potential of $\mathrm{LiC}_{12}$ vs $\mathrm{Li}$ may have been higher than the $\mathrm{Si}$, hence the $\mathrm{LiC}_{12}$ delithiated and the Si was expected to lithiate. Upon transitioning to the graphite phase, which has a lower electrochemical potential vs $\mathrm{Li}$, the potential difference may have crossed over such that the $\mathrm{LiC}_{12}$ then had a lower potential than the $\mathrm{Si}$, hence the Si phase released $\mathrm{Li}$ and lithiated the $\mathrm{LiC}_{12}$ phase at opencircuit. This highlights the importance of accounting for charge equilibration in composite electrodes to access their full capacity during operation upon reaching the cut-off voltage.

The spatial distribution of phases at the beginning and end of the charge process was measured using $1 \mu \mathrm{m}$ resolution XRD-CT that provided sub-particle crystallographic information. At the beginning of the experiment, the cell was in its discharged state with lithiated phases in the graphite/Si electrode. In Figure 5, the spatial distribution of crystalline $\mathrm{Si}$, lithiated $\mathrm{Li}_{x} \mathrm{Si}$, and $\mathrm{LiC}_{12}$ phases is presented. Crystalline $\mathrm{Si}$ cores within lithiated shells were present at the discharged state. The presence of crystalline Si cores indicates that a large portion of the cell's capacity was not being used, reducing the effective energy density of the composite electrode. 
As the Si phase expanded upon lithiation it would have caused internal stresses. Consequently, at the lithiation reaction front, i.e. the interface between the lithiated and non-lithiated phases, compressive stress would have been exerted on the crystalline core during operation. Mechanical stress can retard the reaction rate at the interface, sometimes to the extent that lithiation is arrested, resulting in an nonlithiated, inaccessible, crystalline Si core ${ }^{51}$. From the XRD-CT profiles, residual strain between the lithiated and non-lithiated phases were investigated (see Section 2 and 3 of Supplementary Material). There was no significant residual strain $(>0.05)$ observed within the Si core while the XRD-CT data was being acquired (plots of strain are provided in Figure S15 of Supplementary Material). This is perhaps due to the imaging taking place at open circuit, and the strains being relieved.

The particles presented here were large (10 - $15 \mu \mathrm{m}$ in diameter) relative to more advanced Si-composite electrodes where the Si features are nano-sized ${ }^{52}$. The phase boundary between the $\mathrm{Li}_{x} \mathrm{Si}$ and $\mathrm{Si}$ is distinct in Figure 5, however the resolution was not enough to visualize the formation of sub- $\mu \mathrm{m}$ cracks that could have occurred relative to the position of the phase boundary. However, evidence of delamination is observed which appears to originate from the interface between the crystalline core and amorphous shell (e.g. region 3 in Figure 5b). Whilst evidence for such a two-phase structure has been presented previously $^{52}$ (using TEM) this is a direct observation of its prevalence under relevant conditions (i.e. operating conditions).

Here, the first demonstration of the phase boundary, and its prevalence, being observed inside a relevant operating environment is presented. Most particles that were $<5 \mu \mathrm{m}$ in diameter were observed as lithiated. The lithiated Si shell and crystalline Si core were segmented in the XRD-CT image and their respective diffraction profiles are plotted alongside previously reported metastable crystalline Si phases ${ }^{53-}$ ${ }^{57}$ for comparison. Distinct states of $\mathrm{Li} \mathrm{i}_{\mathrm{x}} \mathrm{Si}$ cannot be distinguished, but it is seen that the diffraction profile of the lithiated shell structure significantly broadens for $Q$ values associated with lithium silicides. The 
shell structure, does not appear to be completely amorphous as extensively reported in literature, but shows characteristics of both crystalline Si and metastable lithium silicides. 

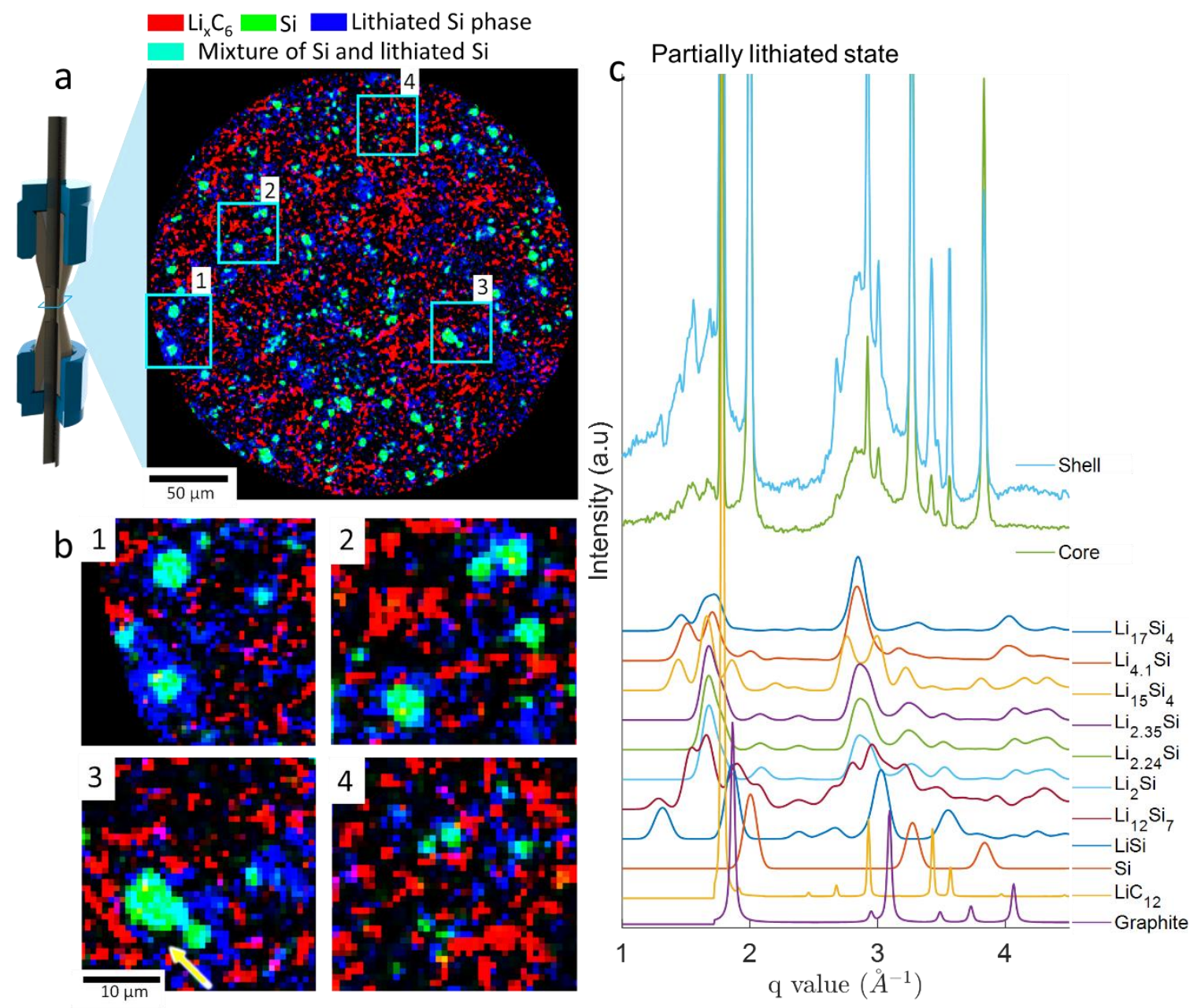

Figure 5. XRD-CT view of the Si-graphite electrode. a) XRD-CT slice taken at the beginning of the charge step, showing a phase distribution map of $\mathrm{LiC}_{12}$ (red), crystalline Si (green) and lithium silicides $\mathrm{Li}_{x} \mathrm{Si}$ (blue). According to additive color mixing, the color teal represents a mixture of green (Si) and blue (lithiated Si). b) magnified regions of interest showing large particles of $\mathrm{Li}_{x} \mathrm{Si}$ phase with crystalline Si cores (1-3) and smaller $\mathrm{Li}_{x} \mathrm{Si}$ particles (4) interspersed in the graphite matrix. The yellow arrow highlights what looks to be evidence of delamination from the crystalline Si core. c) XRD patterns from segmented lithium silicide shells and Si cores from the XRD-CT slice, plotted alongside previously reported lithium silicide phases retrieved from the Inorganic Crystal Structure Database (ICSD). 
Rietveld refinement was carried out on the $\mathrm{LiC}_{12}$ phase (XRD-CT scan at time $=0 \mathrm{~s}$ in Figure $4 \mathrm{a}$ ) and the extracted spatial distribution of lattice parameter is shown in Figure 6a. While in the discharged state, the $\mathrm{LiC}_{12}$ phase was shown to be homogenously lithiated, where the lattice parameter of most of the material was between $7.04 \AA$ and $7.05 \AA$. The graphite particles in the sample were mostly $<10 \mu \mathrm{m}$ in diameter. Figure $6 \mathrm{~b}$ shows magnified views of the particles with sub-particle lattice parameter information. All particles were in the $\mathrm{LiC}_{12}$ phase, but we note the observation of small local differences in lattice parameter - within and between particles. For example, in Figure $6 \mathrm{~b}$, single particles are shown to have regions with lattice parameter values as low as $7.027 \AA$ and as high as $7.055 \AA$. The distribution of lattice parameter from the $\mathrm{Li}_{x} \mathrm{C}$ phases is shown in Figure $6 \mathrm{c}$, where the distribution centers on $7.045 \AA$. 


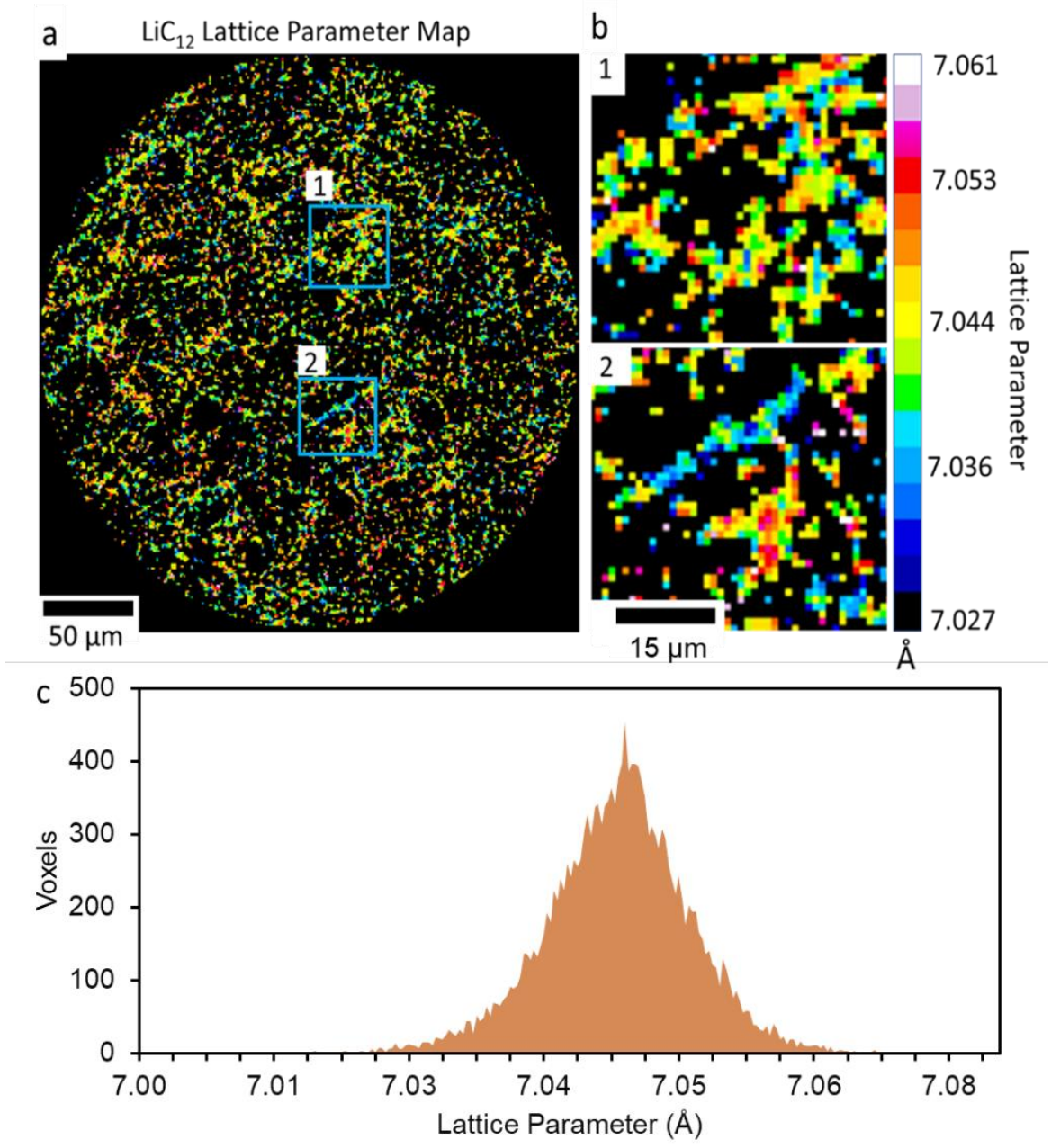

Figure 6. Lattice parameter from XRD-CT of the predominantly $\mathrm{LiC}_{12}$ phase before delithiation. a) Rietveld refined lattice parameter map of the $\mathrm{LiC}_{12}$ phase with (b) magnified regions (1 and 2). c) Histogram showing the distribution of lattice parameter values in the XRD-CT slice.

A final XRD-CT scan was taken after delithiation of the electrode and is shown in Figure 7. The phase map in Figure 7a shows that, as expected, the crystalline Si phase was still present in the electrode following the delithiation process. However, when compared to the core-shell structures observed in Figure 5b, both regions in Figure $7 \mathrm{~b}$ have shrunk. When comparing Figure $5 \mathrm{a}$ to Figure $7 \mathrm{a}$, it is noticeable that some regions are occupied by different phases, e.g. Region 2. As our cell fixture was firmly secured in place, we suspect this discrepancy is likely to have been caused by shifts in the electrode architecture due to the 
shrinkage of active materials upon delithiation, particularly for Si. This caveat should be considered when drawing direct comparisons between the images. By segmenting the core from the shell regions and plotting their respective XRD profiles, the core structures were shown to contain an amalgamation of crystalline Si and lithium silicide phases (Figure 7c). The XRD profile of the core region contains peaks in positions that correspond to some lithium silicides, while also containing distinct peaks that correspond to crystalline Si. The shell region contains further heightened signal from the lithium silicide phases, however the value for $x$ in $\mathrm{Li}_{x} \mathrm{Si}$ is still difficult, if at all possible, to determine. 

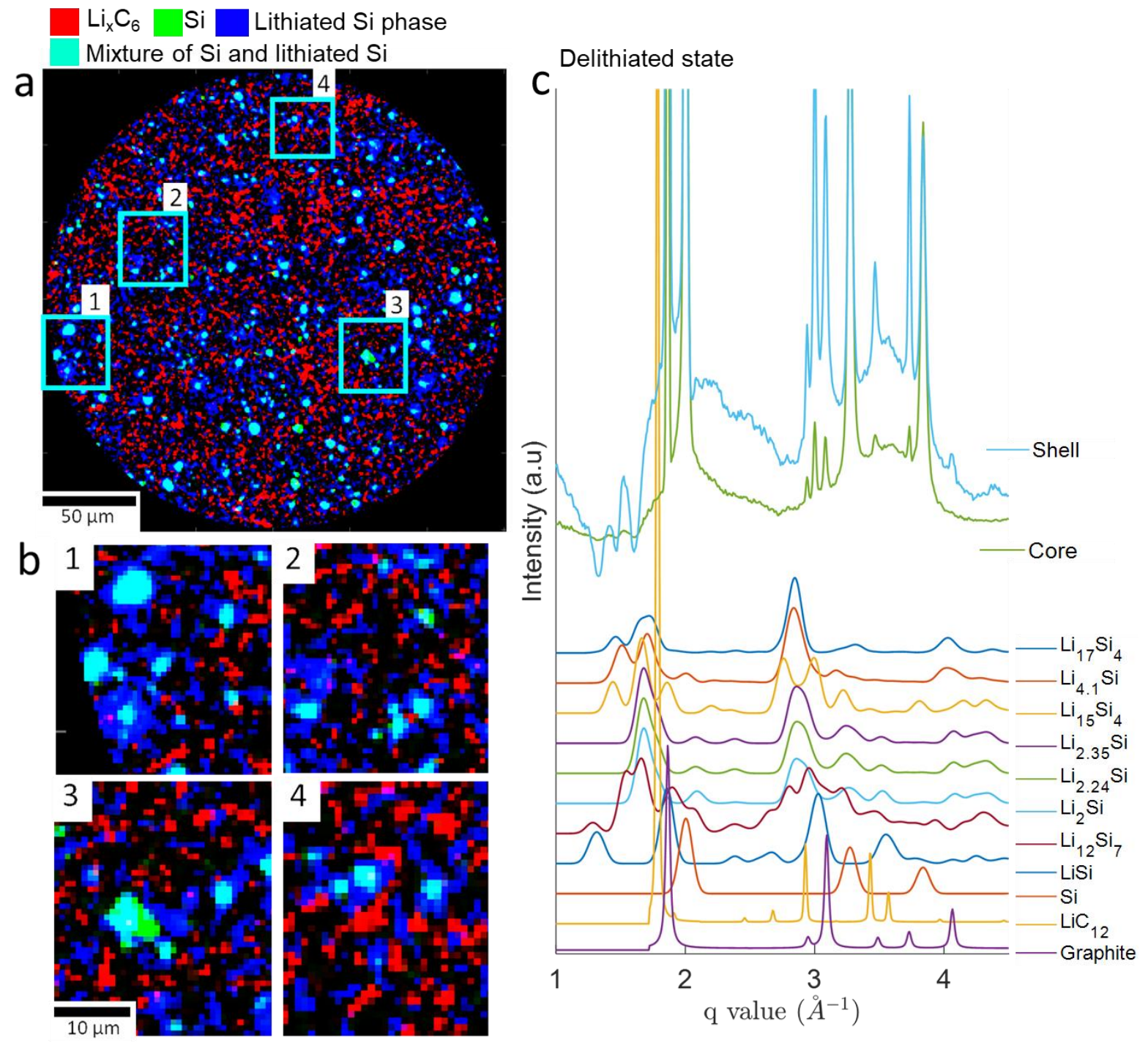

Figure 7. Post-test XRD-CT slice showing the three phases. a) XRD-CT slice taken at the end of the charge step, showing a phase distribution map of graphite (red), crystalline Si (green), and lithium silicide (blue). According to additive color mixing, the color teal represents a mixture of green (Si) and blue (lithiated Si). b) Magnified regions showing the core-shell structure of the Si particles in a delithiated state. c) XRD patterns from segmented lithium silicide shells and Si cores from the XRD-CT slice, plotted alongside previously reported lithium silicide phases retrieved from the Inorganic Crystal Structure Database (ICSD). 
The $\mathrm{LiC}_{12}$ phase had transitioned to the graphite phase and hence Rietveld refinement was carried out using crystallographic parameters from graphite. The resulting spatial distribution of graphite lattice parameters is presented in Figure 8. When compared to the lattice parameter distribution in the discharged state, it was observed that the lithiation state of graphite particles was similarly heterogeneous, with regions exhibiting values of $6.740 \AA$ while the bulk of material is around $6.715 \AA$. The exact cause of such heterogeneity is unknown, however it might have been related to the lithiation equilibration observed in Figure 4c, where some graphite particles were in good contact with lithiated $\mathrm{Si}$ that was at a higher electrochemical potential and thus could have supplied Li ions at open circuit to the graphite phase, thus increasing the lattice parameter of the receiving graphite. As Raynier et al. suggested, the potential difference between graphite at stages 1 and 2 may not be enough to drive equilibration within graphite electrodes, even after one day at open circuit ${ }^{37}$. The potential difference in the case of Reynier's experiments would have been greater than that observed between the Li-C phase here, hence the open-circuit dynamics observed in Figure 4 are expected to have been driven by the potential difference between the $\mathrm{Li}_{x} \mathrm{Si}$ and $\mathrm{Li}-\mathrm{C}$ phases. As this data consists of a single slice, it does not provide information on what lies above and below the particles in view, and has limited resolution, we cannot conclusively state that charge transfer between the lithiated Si and graphite is the definitive cause of the heterogeneous lithiation. We cannot rule out, for example, that the highly lithiated regions stem from heterogeneities in ionic or electronic transport within the electrode. This interaction, and hypothesis on the cause of lithiation heterogeneities in composite electrodes requires further investigation. 


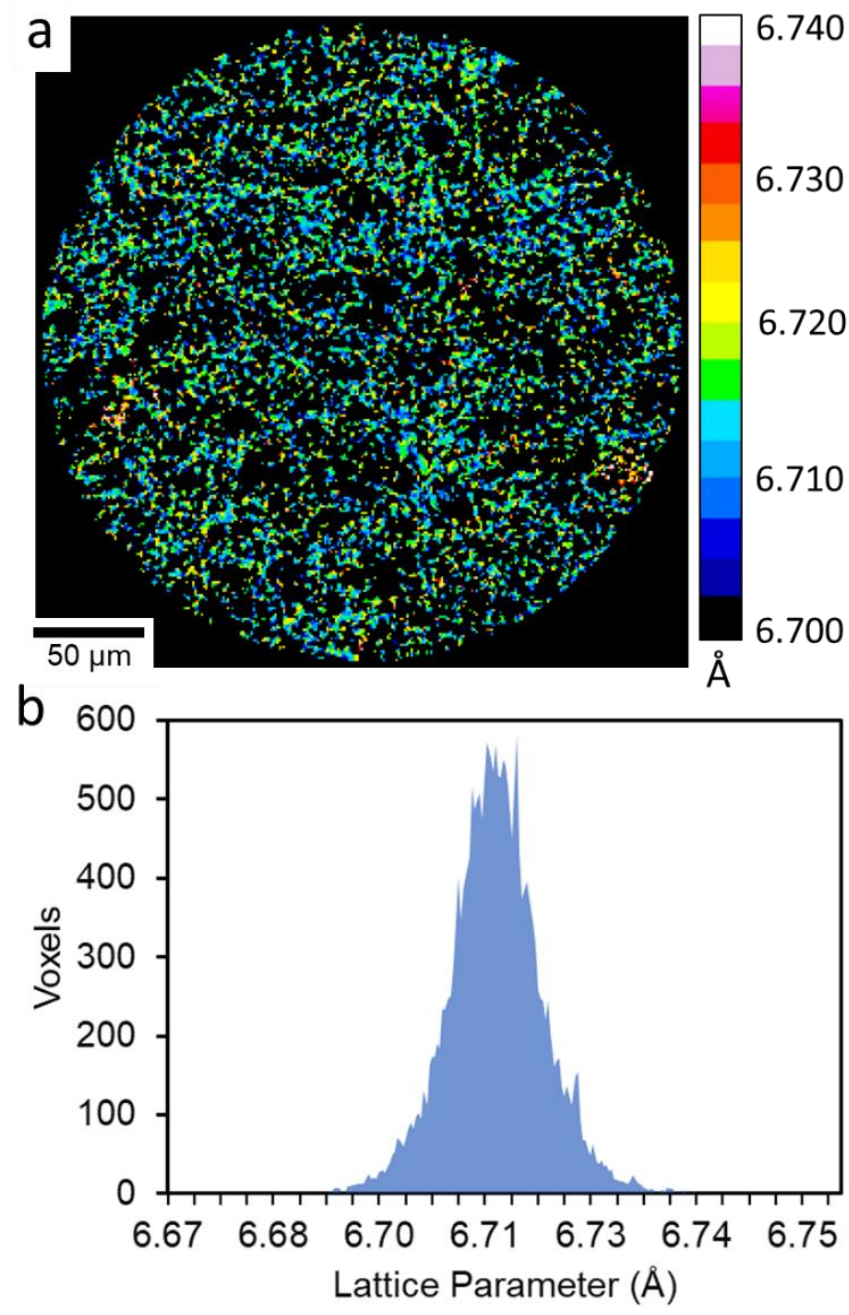

Figure 8. Lattice parameter from XRD-CT of the graphite phase after delithiation. a) Rietveld refined lattice parameter map of the graphite solid-solution phase, and b) histogram showing the distribution of lattice parameter values in the XRD-CT slice.

The cutting-edge capabilities of synchrotron X-ray sources now facilitate spatially-resolved crystallographic investigations of Li-ion battery electrodes with micrometer spatial resolution and down to $0.01 \mathrm{~s}$ temporal resolution. This high spatial and temporal capability was demonstrated to enable identification of heterogeneities in the bulk electrode, as well as within individual particles, in an operando cell environment. High-speed XRD and $1 \mu \mathrm{m}$-resolution XRD-CT, were combined to probe structural dynamics within a composite Si-graphite electrode and to quantify spatial lithiation heterogeneities in 3D. 
This imaging technique was applied to explore charge-balancing kinetics that occur following the transition from operation to open-circuit, as well as to map the utilization of active Si and graphite material during operation. Significant charge balancing between the Si and graphite phases was found to occur following the transition to open-circuit, demonstrating the importance of accounting for different electrochemical potentials of distinct materials in composite electrodes for optimum electrode performance. Using XRD-CT to study crystallographic phases in 3D, it was found that Si particles with a diameter $>5 \mu \mathrm{m}$ had a propensity to form a core-shell structure of non-lithiated crystalline Si and a lithiated lithium silicide phase, respectively. This is likely to have been caused by stressed-induced retardation of the reaction front during lithiation, where lithiated regions expand and compress the $\mathrm{Si}$ core causing high strain during operation at the interface between the lithium silicide phase and nonlithiated crystalline core ${ }^{51}$. However, we did not observe any statistically significant strain $(>0.05)$ nor changes in the peak positions or peak shape of the Si diffraction profiles at open circuit. The core and shell regions were segmented, and their respective summed XRD-profiles investigated, showing that the shell consisted of an amalgamation of metastable lithium silicide phases, and in a delithiated state the core also took on a mixed state of crystalline silicon and metastable lithium silicide. Spatial heterogeneities in the lithiation of graphite were also identified, which when combined with heterogeneities in lithiation of the Si phase, indicates severe under-utilization of the electrode's capacity. The resolution here was not enough to identify, with confidence, the formation of cracks, however some synchrotron sources are capable of combining high-resolution X-ray CT with XRD-CT, which could be used to further elucidate the relationship between the evolving phase boundary between $\mathrm{Si}$ and $\mathrm{Li}_{\mathrm{x}} \mathrm{Si}$ and its propensity to crack at specific locations. Relationships between heterogeneities in lithiation rates, within the bulk electrode and single particles, and degradation mechanisms such as cracking for a plethora of operating conditions, could be elucidated. This will be the topic of future studies. 


\section{Experimental}

\section{Electrode manufacture}

A composite Si-graphite electrode batch was manufactured. The electrode consisted $73 \mathrm{wt} \% \mathrm{Hitachi}$ MAGE graphite, 15 wt\% Si, 10 wt\% PAA binder and 2 wt\% Super P carbon. Disks of electrode with a diameter of $12.7 \mathrm{~mm}$ were prepared for implanting into coin cells. Two coin cells were prepared and tested. The Si-graphite electrode had a measured capacity of ca. $5 \mathrm{mAh}$, corresponding to $c a .0 .04$ $\mathrm{mAh} / \mathrm{mm}^{2}$.

\section{Operando micro-cell}

For XRD-CT experiments, a bespoke cell of Li vs Si-graphite composite electrode was manufactured. The cell holder consisted in part (nuts and ferrules) of a 1/8" $(3.175 \mathrm{~mm})$ PFA Swagelok union and is shown in Figure 2. A custom PEEK holder housed the cell (beige component in Figure 2). The current collecting pins were made from 316 stainless steel. A $1 \mathrm{~mm}$ disk of Si-graphite electrode on a Cu current collector was punched and layered flat on top of the negative pin. The $1 \mathrm{~mm}$ disk is estimated to have a capacity of $0.0314 \mathrm{mAh}$ (at $0.04 \mathrm{mAh} / \mathrm{mm}^{2}$ ). An electrolyte wetted borosilicate glass fiber separator (Whatmann GFD grade, GE) was used, and a counter electrode of Li metal was compressed onto the positive pin which was then inserted into the assembly displacing excess electrolyte as it was pushed in. Electrically conducting wires were secured against the current collecting pins by tightening a collar-screw (shown in Figure 2a).

\section{High-speed operando XRD and XRD-CT}

A monochromatic beam of $50 \mathrm{keV}(0.2480 \AA)$ was used for all diffraction measurements. The micro-cell was secured into a gas delivery stub, itself mounted in a standard goniometer, as shown in Figure 2 . The goniometer was fixed to a rotation stage set upon a translation stage to facilitate the movements required 
for the CT measurement. The beam was focused to $1 \mu \mathrm{m}$ using focusing Kirkpatrick-Baez mirrors, and a collimator was placed directly on front of the sample to remove background. A high-energy single photoncounting Pilatus 3 X CdTe $2 \mathrm{M}$ detector was used for recording the diffracted signal. An XRD point-scan was recorded at a rate of $0.1 \mathrm{~Hz}$ during operation. XRD point scans were recorded at $100 \mathrm{~Hz}$ for $15 \mathrm{~s}$ following open circuit, and at $10 \mathrm{~Hz}$ for a further 16 mins. The detector calibration was performed using a $\mathrm{CeO}_{2} \mathrm{NIST}$ standard. Every 2D diffraction image was converted to a 1D powder diffraction pattern after applying a $10 \%$ trimmed mean filter to remove outliers using the nDTomo and pyFAl software packages ${ }^{58-60}$. The data integration was performed with a fast GPU processing. The final XRD-CT images (i.e. reconstructed data volume) were reconstructed using the filtered back projection algorithm.

XRD-CT slices of the cell were recorded when the cell was fully charged and discharged. The XRD-CT scan consisted of two scans; a coarse scan that captured the entire width of the assembly including the PEEK holder, and a region of interest (ROI) $1 \mu \mathrm{m}$-resolution scan. The coarse scan involved 71 rastering points with step sizes of $50 \mu \mathrm{m}$, and 50 rotations at angular increments of 3 degrees (completing a 180 rotation). The ROI scan consisted of 301 rastering point measurements with step sizes of $1 \mu \mathrm{m}$, and 300 rotations at angular increments of 0.6 degrees. The coarse scan was used for correction of the ROI scan to subtract signal from external entities such as the PEEK casing and electrode material outside of the field of view.

\section{Data processing and Rietveld refinement}

A detailed description of this process is provided in Section 1 of Supplementary Material. Here, a brief summary is provided. A mask was applied to the coarse XRD-CT dataset in order to extract a diffraction pattern containing only signal generated by the PEEK component of the sample. LeBail whole powder profile analysis of the PEEK diffraction pattern was performed using a Pbcn space group. This refined PEEK model was used to fit the PEEK scattering signal in the point XRD measurements. The model also included

$\mathrm{Si}(\mathrm{Fd}-3 \mathrm{mS}), \mathrm{Cu}(\mathrm{Fm}-3 \mathrm{~m}), \mathrm{LiC}_{12}(\mathrm{P} 6 / \mathrm{mmm})$ and graphite $(\mathrm{P} 6 / \mathrm{mmm})$. LeBail analysis was performed for the 
PEEK signal while Rietveld analysis was performed for the $\mathrm{Si}, \mathrm{Cu}, \mathrm{LiC}_{12}$ and graphite. For the $1 \mu \mathrm{m}$ resolution region-of-interest XRD-CT data, the PEEK signal is absent, and the model included only $\mathrm{Si}, \mathrm{Cu}$, $\mathrm{LiC}_{12}$ and graphite. In both cases, the quality of the fit is very good (see Section 1 of Supplementary Material). Apart from the background and the scale factors for all phases, the other parameters refined during the Rietveld analysis of the diffraction data were the lattice parameters $a$ and $c$ for $\mathrm{LiC}_{12}$ and graphite. Here, the lattice constant $c$ for the graphite and $\mathrm{LiC}_{12}$ phase was graphed, as it showed the most significant change during (de)lithiation. The analysis of the diffraction data was performed with the Topas v5 software ${ }^{61}$. The core-shell structures of the Si particles were decoupled using MATLAB and their respective XRD profiles were summed and plotted (see Section 3 of Supplementary Material).

\section{Acknowledgements}

This work was authored by the National Renewable Energy Laboratory managed and operated by Alliance for Sustainable Energy, LLC for the U.S. Department of Energy (DOE) under Contract No. DE-AC3608GO28303. Funding provided by the U.S. Department of Energy Vehicle Technology Office. The research is supported by the Vehicle Technologies Office of the U.S. Department of Energy Office of Energy Efficiency and Renewable Energy, under the supervision of David Howell (Manager), and Brian Cunningham and Peter Faguy (Technology Managers). The views expressed in the article do not necessarily represent the views of the DOE or the U.S. Government. The U.S. Government and the publisher, by accepting the article for publication, acknowledges that the U.S. Government retains a nonexclusive, paid-up, irrevocable, worldwide license to publish or reproduce the published form of this work, or allow others to do so, for U.S. Government purposes. Antonis Vamvakeros is supported (in part) through funding received from the European Union Horizon 2020 research and innovation programme under Grant Agreement No. 679933 (MEMERE project). The authors would like to acknowledge the ISCF 
Faraday Challenge Fast Start project on "Degradation of Battery Materials" made available through grant EP/S003053/1. PRS acknowledges funding from the Royal Academy of Engineering for financial support under the Chair in Emerging Technologies scheme. These experiments were performed at beamline ID15A at the ESRF (Grenoble, France). We are grateful to the ESRF for granting beamtime on beamline ID15A.

Author contributions: D.P.F conceived, prepared, carried out the experiments and wrote the paper. A.V. carried out the reconstruction of the of the XRDCT data and Rietveld refinement of the XRD data. D.P.F., L.C., and C.B. designed and prepared the composite electrodes and cells for X-ray analysis. L.C., D.P.F., C.T., T.M.M.H., M.D.M. and S.D. assisted with experiments during the beamtime. S.J., A.M.B., and A.V. created the software for XRDCT reconstruction and interpretation. M.D.M prepared the beamline and beam conditions for the experiments. K.S., C.B., D.J.L.B., and P.R.S. provided valuable advice and guidance for preparing the experiments and interpreting the results.

Declaration: The authors declare no competing financial interest.

Supporting Information available online.

\section{References}

1. Harris, S. J.; Lu, P. The Journal of Physical Chemistry C 2013, 117, (13), 6481-6492.

2. Qi, Y.; Harris, S. J. Journal of The Electrochemical Society 2010, 157, (6), A741-A747.

3. Wang, J.; Karen Chen-Wiegart, Y.-C.; Eng, C.; Shen, Q.; Wang, J. Nature Communications 2016, 7, 12372.

4. Nelson Weker, J.; Li, Y.; Shanmugam, R.; Lai, W.; Chueh, W. C. ChemElectroChem 2015, 2, (10), 1576-1581.

5. $\quad$ Chao, S.-C.; Yen, Y.-C.; Song, Y.-F.; Chen, Y.-M.; Wu, H.-C.; Wu, N.-L. Electrochemistry Communications 2010, 12, (2), 234-237.

6. Mao, Y.; Wang, X.; Xia, S.; Zhang, K.; Wei, C.; Bak, S.; Shadike, Z.; Liu, X.; Yang, Y.; Xu, R.; Pianetta, P.; Ermon, S.; Stavitski, E.; Zhao, K.; Xu, Z.; Lin, F.; Yang, X.-Q.; Hu, E.; Liu, Y. Advanced Functional Materials 2019, 0, (0), 1900247.

7. Nanda, J.; Remillard, J.; O'Neill, A.; Bernardi, D.; Ro, T.; Nietering, K. E.; Go, J.-Y.; Miller, T. J. Advanced Functional Materials 2011, 21, (17), 3282-3290. 
8. $\quad$ Ali, I.; Tippabhotla, S. K.; Radchenko, I.; Al-Obeidi, A.; Stan, C. V.; Tamura, N.; Budiman, A. S. Frontiers in Energy Research 2018, 6, 19.

9. Liu, J.; Kunz, M.; Chen, K.; Tamura, N.; Richardson, T. J. The Journal of Physical Chemistry Letters 2010, 1, (14), 2120-2123.

10. Harris, S. J.; Timmons, A.; Baker, D. R.; Monroe, C. Chemical Physics Letters 2010, 485, (4), 265-

274.

11. Yao, K. P. C.; Okasinski, J. S.; Kalaga, K.; Shkrob, I. A.; Abraham, D. P. Energy \& Environmental Science 2019, 12, (2), 656-665.

12. Eastwood, D. S.; Yufit, V.; Gelb, J.; Gu, A.; Bradley, R. S.; Harris, S. J.; Brett, D. J. L.; Brandon, N. P.; Lee, P. D.; Withers, P. J.; Shearing, P. R. Advanced Energy Materials 2014, 4, (4).

13. Finegan, D. P.; Tudisco, E.; Scheel, M.; Robinson, J. B.; Taiwo, O. O.; Eastwood, D. S.; Lee, P. D.; Di Michiel, M.; Bay, B.; Hall, S. A.; Hinds, G.; Brett, D. J. L.; Shearing, P. R. Advanced Science 2016, 3, (3).

14. Ulvestad, A.; Cho, H. M.; Harder, R.; Kim, J. W.; Dietze, S. H.; Fohtung, E.; Meng, Y. S.; Shpyrko, O. G. Applied Physics Letters 2014, 104, (7), 073108.

15. Ulvestad, A.; Singer, A.; Cho, H.-M.; Clark, J. N.; Harder, R.; Maser, J.; Meng, Y. S.; Shpyrko, O. G. Nano Letters 2014, 14, (9), 5123-5127.

16. Ulvestad, A.; Clark, J. N.; Singer, A.; Vine, D.; Cho, H. M.; Harder, R.; Meng, Y. S.; Shpyrko, O. G. Physical Chemistry Chemical Physics 2015, 17, (16), 10551-10555.

17. Ebner, M.; Marone, F.; Stampanoni, M.; Wood, V. Science 2013, 342, (6159), 716-720.

18. Wang, J.; Eng, C.; Chen-Wiegart, Y.-C. K.; Wang, J. Nature Communications 2015, 6, 7496.

19. Paz-Garcia, J. M.; Taiwo, O. O.; Tudisco, E.; Finegan, D. P.; Shearing, P. R.; Brett, D. J. L.; Hall, S. A. Journal of Power Sources 2016, 320, 196-203.

20. Pietsch, P.; Hess, M.; Ludwig, W.; Eller, J.; Wood, V. Scientific Reports 2016, 6, 27994.

21. Lim, J.; Li, Y.; Alsem, D. H.; So, H.; Lee, S. C.; Bai, P.; Cogswell, D. A.; Liu, X.; Jin, N.; Yu, Y.-S.;

Salmon, N. J.; Shapiro, D. A.; Bazant, M. Z.; Tyliszczak, T.; Chueh, W. C. Science 2016, 353, (6299), 566-

571.

22. Dahn, J. R. Physical Review B 1991, 44, (17), 9170-9177.

23. Satoh, A.; Takami, N.; Ohsaki, T. Solid State lonics 1995, 80, (3), 291-298.

24. Yao, K. P. C.; Okasinski, J. S.; Kalaga, K.; Almer, J. D.; Abraham, D. P. Advanced Energy Materials 2019, 9, (8), 1803380.

25. Sasaki, T.; Villevieille, C.; Takeuchi, Y.; Novák, P. Advanced Science 2015, 2, (7), 1500083.

26. Harding, G.; Kosanetzky, J.; Neitzel, U. Medical Physics 1987, 14, (4), 515-525.

27. Sottmann, J.; Di Michiel, M.; Fjellvåg, H.; Malavasi, L.; Margadonna, S.; Vajeeston, P.; Vaughan, G. B. M.; Wragg, D. S. Angewandte Chemie International Edition 2017, 56, (38), 11385-11389.

28. Jensen, K. M. Ø.; Yang, X.; Laveda, J. V.; Zeier, W. G.; See, K. A.; Michiel, M. D.; Melot, B. C.; Corr, S. A.; Billinge, S. J. L. Journal of The Electrochemical Society 2015, 162, (7), A1310-A1314.

29. Bleuet, P.; Welcomme, E.; Dooryhée, E.; Susini, J.; Hodeau, J.-L.; Walter, P. Nature Materials 2008, 7, 468.

30. Bonnin, A.; Wright, J. P.; Tucoulou, R.; Palancher, H. Applied Physics Letters 2014, 105, (8), 084103

31. Jacques, S. D. M.; Di Michiel, M.; Beale, A. M.; Sochi, T.; O'Brien, M. G.; Espinosa-Alonso, L.; Weckhuysen, B. M.; Barnes, P. Angewandte Chemie International Edition 2011, 50, (43), 10148-10152.

32. Vamvakeros, A.; Jacques, S. D. M.; Di Michiel, M.; Matras, D.; Middelkoop, V.; Ismagilov, I. Z.; Matus, E. V.; Kuznetsov, V. V.; Drnec, J.; Senecal, P.; Beale, A. M. Nature Communications 2018, 9, (1), 4751.

33. Su, X.; Wu, Q.; Li, J.; Xiao, X.; Lott, A.; Lu, W.; Sheldon, B. W.; Wu, J. Advanced Energy Materials 2014, 4, (1), 1300882. 
34. Hassan, F. M.; Batmaz, R.; Li, J.; Wang, X.; Xiao, X.; Yu, A.; Chen, Z. Nature Communications 2015, 6, 8597.

35. Wetjen, M.; Pritzl, D.; Jung, R.; Solchenbach, S.; Ghadimi, R.; Gasteiger, H. A. Journal of The Electrochemical Society 2017, 164, (12), A2840-A2852.

36. Tanida, H.; Yamashige, H.; Orikasa, Y.; Gogyo, Y.; Arai, H.; Uchimoto, Y.; Ogumi, Z. The Journal of Physical Chemistry C 2016, 120, (9), 4739-4743.

37. Reynier, Y.; Yazami, R.; Fultz, B. Journal of Power Sources 2007, 165, (2), 616-619.

38. Rashid, M.; Gupta, A. Journal of The Electrochemical Society 2015, 162, (2), A3145-A3153.

39. Park, J.; Park, S. S.; Won, Y. S. Electrochimica Acta 2013, 107, 467-472.

40. Harris, S. J.; Rahani, E. K.; Shenoy, V. B. Journal of The Electrochemical Society 2012, 159, (9), A1501-A1507.

41. Migge, S.; Sandmann, G.; Rahner, D.; Dietz, H.; Plieth, W. Journal of Solid State Electrochemistry 2005, 9, (3), 132-137.

42. Tan, C.; Daemi, R. S.; Taiwo, O. O.; Heenan, M. T.; Brett, J. D.; Shearing, R. P. Materials 2018, 11, (11).

43. Borkiewicz, O. J.; Wiaderek, K. M.; Chupas, P. J.; Chapman, K. W. The Journal of Physical Chemistry Letters 2015, 6, (11), 2081-2085.

44. Daumas, N.; Hérold, A. Compt. Rend., Sér. C 1969, 268, 373.

45. Gavilán-Arriazu, E. M.; Pinto, O. A.; López de Mishima, B. A.; Barraco, D. E.; Oviedo, O. A.; Leiva, E. P. M. Electrochemistry Communications 2018, 93, 133-137.

46. Senyshyn, A.; Dolotko, O.; Mühlbauer, M. J.; Nikolowski, K.; Fuess, H.; Ehrenberg, H. Journal of The Electrochemical Society 2013, 160, (5), A3198-A3205.

47. Taminato, S.; Yonemura, M.; Shiotani, S.; Kamiyama, T.; Torii, S.; Nagao, M.; Ishikawa, Y.; Mori, K.; Fukunaga, T.; Onodera, Y.; Naka, T.; Morishima, M.; Ukyo, Y.; Adipranoto, D. S.; Arai, H.; Uchimoto, Y.; Ogumi, Z.; Suzuki, K.; Hirayama, M.; Kanno, R. Scientific Reports 2016, 6, 28843.

48. Woo, K. C.; Mertwoy, H.; Fischer, J. E.; Kamitakahara, W. A.; Robinson, D. S. Physical Review B 1983, 27, (12), 7831-7834.

49. Fong, R.; von Sacken, U.; Dahn, J. R. Journal of The Electrochemical Society 1990, 137, (7), 2009-

2013.

50. Missyul, A.; Bolshakov, I.; Shpanchenko, R. Powder Diffraction 2017, 32, (S1), S56-S62.

51. Zhang, S. npj Computational Materials 2017, 3, (1), 7.

52. Liu, X. H.; Zhong, L.; Huang, S.; Mao, S. X.; Zhu, T.; Huang, J. Y. ACS Nano 2012, 6, (2), 1522-1531.

53. Li, J.; Dahn, J. R. Journal of The Electrochemical Society 2007, 154, (3), A156-A161.

54. Nesper, R.; von Schnering, H. G. Journal of Solid State Chemistry 1987, 70, (1), 48-57.

55. von Schnering, H. G.; Nesper, R.; Curda, J.; Tebbe, K.-F. Angewandte Chemie International Edition in English 1980, 19, (12), 1033-1034.

56. Misra, S.; Liu, N.; Nelson, J.; Hong, S. S.; Cui, Y.; Toney, M. F. ACS Nano 2012, 6, (6), 5465-5473.

57. Key, B.; Bhattacharyya, R.; Morcrette, M.; Seznéc, V.; Tarascon, J.-M.; Grey, C. P. Journal of the American Chemical Society 2009, 131, (26), 9239-9249.

58. Ashiotis, G.; Deschildre, A.; Nawaz, Z.; Wright, J. P.; Karkoulis, D.; Picca, F. E.; Kieffer, J. Journal of Applied Crystallography 2015, 48, (2), 510-519.

59. Vamvakeros, A.; Jacques, S. D. M.; Di Michiel, M.; Middelkoop, V.; Egan, C. K.; Cernik, R. J.; Beale, A. M. Journal of Applied Crystallography 2015, 48, (6), 1943-1955.

60. Kieffer, J.; Petitdemange, S.; Vincent, T. Journal of Synchrotron Radiation 2018, 25, (2), 612-617.

61. Coelho, A. Journal of Applied Crystallography 2018, 51, (1), 210-218. 